\title{
Active-RC Filters Suitable as Antialiasing and/or Reconstruction Filters
}

\author{
Bohumil Brtník \\ Department of Electrical Engineering \\ University of Pardubice \\ Náměstí Čs.Legií 535 \\ 53002 Pardubice Czech Republic \\ bohumil.brtnik@upce.cz
}

\author{
David Matoušek \\ Department of Electrical Engineering \\ University of Pardubice \\ Náměstí Čs.Legií 535 \\ 53002 Pardubice Czech Republic \\ david.matousek@upce.cz
}

\begin{abstract}
The discrete time signal processing circuit requires an anti-aliasing filter at the input and a reconstruction filter at the output, generally. In this paper, selected basic structures of biquads are described and compared with a view to the degradation of the attenuation over the transient frequency of the operational amplifier. Concretely, low-pass and bandpass Sallen-Key, Huelsman (i.e. multi feedback) and non-cascade filter structures are confronted with the limits of a real operational amplifier. Firstly, the reasons for the degradation of the attenuation are explained theoretically. Secondly, these conclusions are verified by simulations. These simulations were performed by spicelike circuit simulator MicroCap version 11.
\end{abstract}

Keywords-low-pass filter structure; band-pass filter structure; real operational amplifier; frequency response; decreasing of the attenuation at the high frequencies

\section{INTRODUCTION}

The discrete-time signal processing circuit i.e. switched capacitor circuits, switched currents and digital signal processing circuits, require an antialiasing filter at the input and a reconstruction filter at the output [1], [2], [3] for their operation. These filters should have low-pass filter properties, but the circuit realization based on an active RC (ARC) filter contains the operational amplifiers with real properties, thus these limit factors modify the original monotone-decreasing frequency response to a nonmonotonic character.

The main disadvantage of the all discrete-time signal processing circuits is the periodicity of its frequency response, as is depicted in Fig. 1. Therefore, the main task of the reconstruction filter must be not only decreasing of the noise invoked by the digital-to-analogue conversion settling, but also the attenuation of frequencies upper half of sampling frequency. The realization of these filters can use active RC filters, where an active element can be an operational amplifier, transconductance amplifier and/or transimpedance amplifier.

In the following text, the usage of the real operational amplifier in basic filter structures will be discussed. Because of the smallest sensitivity to altering the change elements parameters, higher order filters are assembled from the second-order partial filters (so-called biquads) and/or third-order filters.

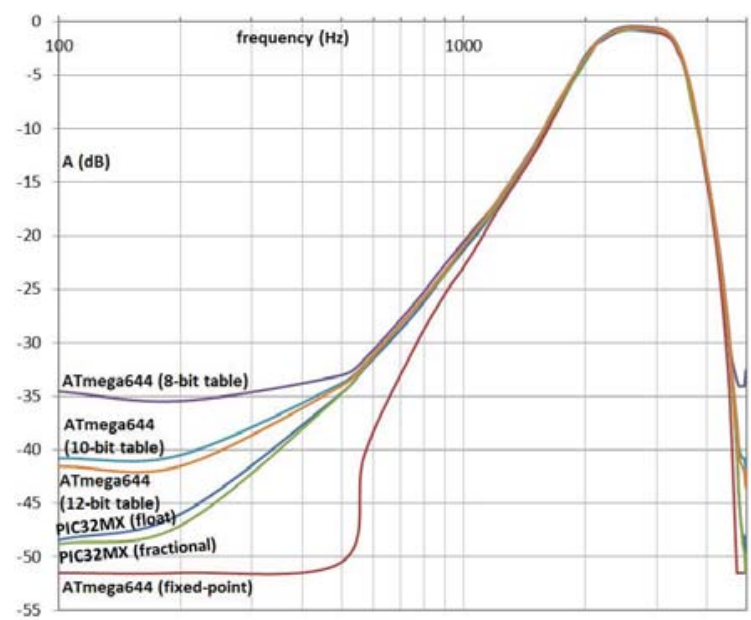

Figure 1. Digital filter attenuation versus format data word.

Some biquad filters structures are characterized by a degradation of the attenuation at high frequencies [4], [5]. This degradation of the attenuation occurs only for some filters of the even orders, i.e. for the biquads, and is caused by the final value of the output resistance of the used operational amplifier [6] (see Fig. 2).
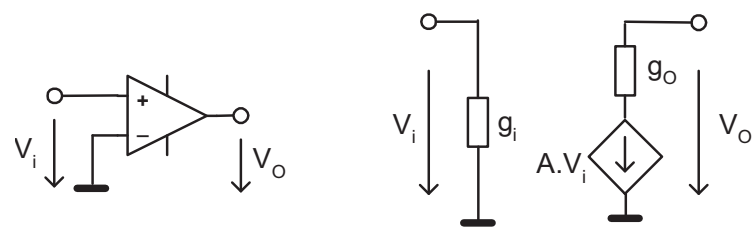

Figure 2. Real operational amplifier and its model.

The reason of these attenuation losses is depicted in Fig. 3 for a second-order low-pass filter. The ideal course of the second-order low-pass filter frequency response is a monotonous decreasing of the magnitude value over the cutoff frequency $\omega_{0}$ at whole frequency range with $-40 \mathrm{~dB} / \mathrm{dec}$ slope. The finite value $\omega_{T}$ of the open loop gain $A_{0}$ of the used operational amplifier leads to the break of this ideal slope. The result is a loss of attenuation in the stopband for the frequencies around the transient frequency of the used operational amplifier $\omega_{\mathrm{T}}$. 


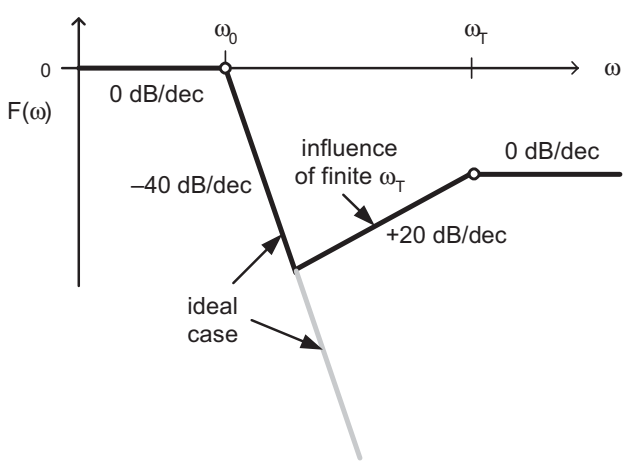

Figure 3. A frequency response of the low-pass biquad.

\section{BASIC FILTERS STRUCTURE}

Filter characteristics in the transition area between pass-band and stop-band frequencies are described in the generally available literature.

\section{A. LP Sallen-Key Structure of Even Order}

The Sallen-Key low-pass filter i.e. LP-SK [7] using an operational amplifier as a voltage follower exhibit the lowest sensitivity to the passive elements, smaller than the involvement of Huelsmann low-pass filter structure i.e. LP-H [8], [9]. Therefore, from the sensitivity of the filter viewpoint, the composition from biquads LP-SK is the best. Commonly used filter structure is shown in Fig. 4.

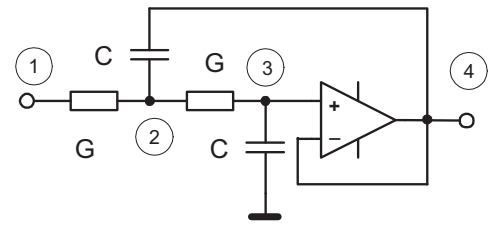

Figure 4. The structure of common biquad LP-SK.

The main disadvantage of LP-SK biquad filters for odd orders, however, is a reduction of the attenuation in the stop-band. We know that the amplification factor A of an operational amplifier is reduced to zero at high frequencies. The reactance of capacitors nears to zero for frequencies close to infinite. The voltage ratio has a nonzero value in the zone over of transient frequency of the operational amplifier [9], as shown in the simulation result in Fig. 5, this fact explains the degradation of the filter properties.



Figure 5. Simulation result of common biquad LP-SK.

For commonly used operational amplifier of type LM741 with following parameters $\operatorname{R}_{\text {OUTAC }}=50 \Omega$, $\mathrm{A}=200 \mathrm{~V} / \mathrm{mV}, \quad \mathrm{GBW}=1 \mathrm{MHz}, \quad$ we see that attenuation is reduced to $50 \mathrm{~dB}$ after reaching $55 \mathrm{~dB}$ attenuation.

Thus, the filter attenuation in the stop-band [6] does not increase to infinity, but a nonzero output voltage $\mathrm{V}_{4}$ is determined by (1).

$$
\mathrm{V}_{4}=\mathrm{V}_{1} \cdot \frac{\frac{\mathrm{R} \cdot \mathrm{r}_{\mathrm{i}} \cdot \mathrm{r}_{\mathrm{o}}}{\mathrm{R} \cdot \mathrm{r}_{\mathrm{i}}+\mathrm{R} \cdot \mathrm{r}_{\mathrm{o}}+\mathrm{r}_{\mathrm{i}} \cdot \mathrm{r}_{\mathrm{o}}}}{\mathrm{R}+\frac{\mathrm{R} \cdot \mathrm{r}_{\mathrm{i}} \cdot \mathrm{r}_{\mathrm{o}}}{\mathrm{R} \cdot \mathrm{r}_{\mathrm{i}}+\mathrm{R} \cdot \mathrm{r}_{\mathrm{o}}+\mathrm{r}_{\mathrm{i}} \cdot \mathrm{r}_{\mathrm{o}}}}>0
$$

where: $r_{i}$ - input resistance, $r_{o}-$ output resistance of the op. amp. and $\mathrm{R}$ - resistance of the working resistor in the filter, where:

$$
\mathrm{r}_{\mathrm{i}}=\frac{1}{\mathrm{~g}_{\mathrm{i}}}, \mathrm{r}_{\mathrm{O}}=\frac{1}{\mathrm{~g}_{\mathrm{o}}}, \mathrm{R}=\frac{1}{\mathrm{G}}
$$

The reason for this effect [7] is a feedback capacitor as is shown in the equivalent circuit in Fig. 6.

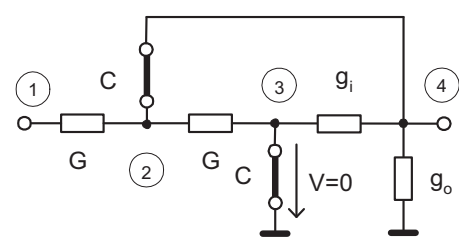

Figure 6. Model of the LP-SK biquads in high frequencies.

\section{B. LP Sallen-Key Structure of Odd Order}

For the construction of odd-numbered filters, we extend Sallen-Key biquad about a passive RC net. The resulting structure contains three $\mathrm{RC}$ members, i.e. this is a third-order structure. Its schematic diagram is depicted in Fig. 7.

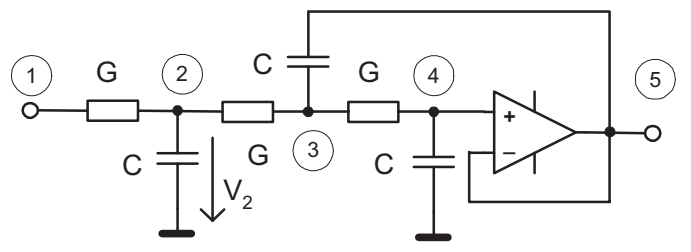

Figure 7. The third-order structure of LP-SK.

Figure 8 is a simplified schematic diagram of the circuit from Fig. 7 at high frequencies (all capacitors are substituted to a short circuit).

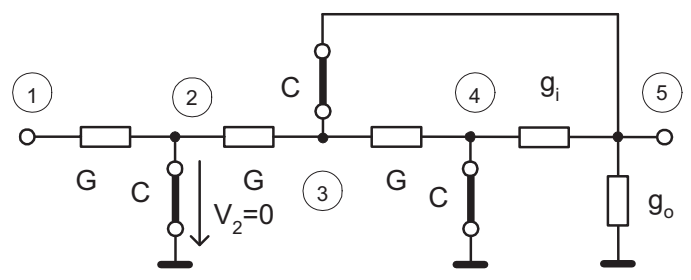

Figure 8. Model of the third order structure of LP-SK structure in high frequencies.

The voltage $V_{2}$ at the node 2 is equal to zero, therefore the second part of the filter is excited by this null voltage, thus output voltage must be equal to zero, 
too. The magnitude of the voltage transfer ratio at the highest frequencies is equal to zero (3).

$$
\mathrm{F}_{\text {inf }}=\lim _{\omega \rightarrow \infty} \frac{\mathrm{V}_{\text {OUT }}}{\mathrm{V}_{\text {IN }}}=0
$$

where: $F_{\text {inf }}, V_{\text {IN }}, V_{\text {OUT }}$ were specified above.

Simulation by program MicroCap version 11 verified this calculated solution (see Fig. 9).

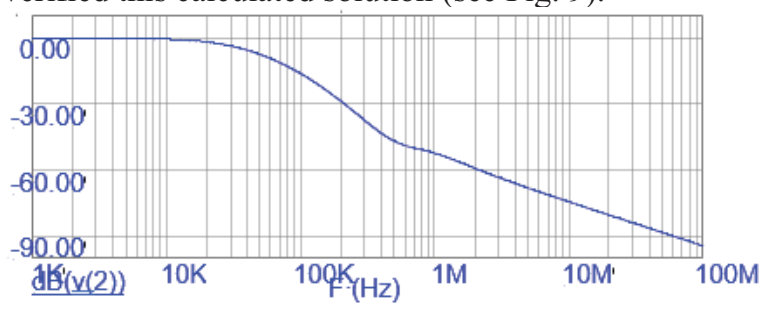

Figure 9. Simulation result of LP third order SK structure at the highest frequencies.

\section{LP Huelsmann Structure}

Now, we focus on the simplest LP-H (LP-MFB) filter [7], [8] at the highest frequencies. Fig. 10 is a schematic diagram of a biquad $(G, C$ and $C$ are values of conductance and capacitance of used resistors and capacitors).

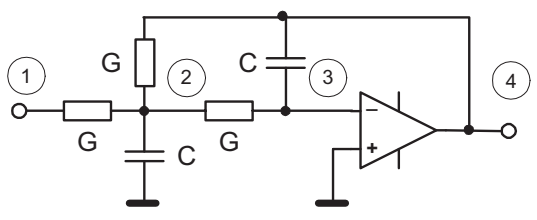

Figure 10. LP biquad Huelsmann (MFB) structure.

Figure 11 is a simplified schematic diagram of the biquad at high frequencies (all capacitors are substituted to a short circuit).

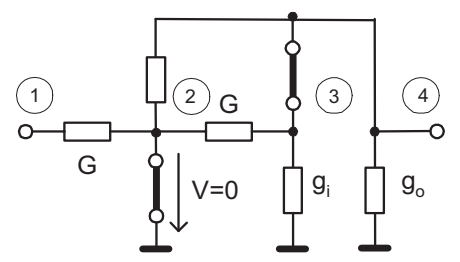

Figure 11. Low-pass biquad Huelsmann structure at the highest frequencies.

At high frequencies, the operational amplifier loses its amplification factor, now. The voltage at node \#2 is equal to zero, therefore the second part of the filter is excited by zero voltage, thus output voltage must be equal to zero, too. The magnitude of the voltage transfer ratio at the highest frequencies is equal to zero (4):

$$
\mathrm{F}_{\text {inf }}=\lim _{\omega \rightarrow \infty} \frac{\mathrm{V}_{\text {OUT }}}{\mathrm{V}_{\text {IN }}}=0
$$

where: $F_{\text {inf }}, V_{\text {IN }}, V_{\text {OUT }}$ were specified above.

Simulation by program MicroCap version 11 verified this calculated solution. Opamp LF155 $(G B P=2.5 \mathrm{MHz})$ was used for these simulations. The degradation of the attenuation at high frequencies did not occur for output corresponded to output node 4 (see Fig. 12).

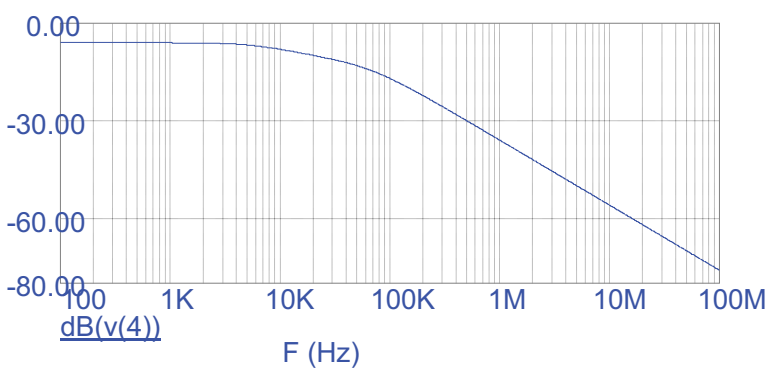

Figure 12. Simulation results LP-H at the highest frequencies.

\section{Non-Cascade Structure}

The non-cascading filter structure [10] is used when the output signal contains a DC component. A resistor is connected between input and output nodes, the frequency variable impedance is realized by a circuit containing an operational amplifier, which is connected between the output node and ground. The schematic diagram of the simplest non-cascading filter is depicted in Fig. 13.



Figure 13. The structure of the non-cascade filter.

Figure 14 is a simplified schematic diagram of the biquad at high frequencies (where all capacitors are substituted to a short circuit).

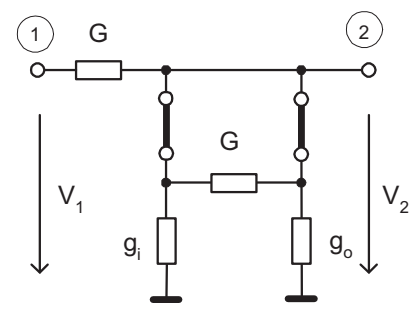

Figure 14. Model of the non-cascade filter in high frequencies.

As we can see from Fig. 14, the filter attenuation in the stop-band [6] (i.e. at high frequencies) does not increase to infinity, but nonzero output voltage $V_{2}$ is determined by (5).

$$
\mathrm{V}_{2}=\frac{\frac{\mathrm{r}_{\mathrm{i}} \cdot \mathrm{r}_{0}}{\mathrm{r}_{\mathrm{i}}+\mathrm{r}_{0}}}{\mathrm{R}+\frac{\mathrm{r}_{\mathrm{i}} \cdot \mathrm{r}_{0}}{\mathrm{r}_{\mathrm{i}}+\mathrm{r}_{0}}} \cdot \mathrm{V}_{1}>0
$$


Simulation by program MicroCap version 11 verified this calculated solution. The result is depicted in Fig. 15.

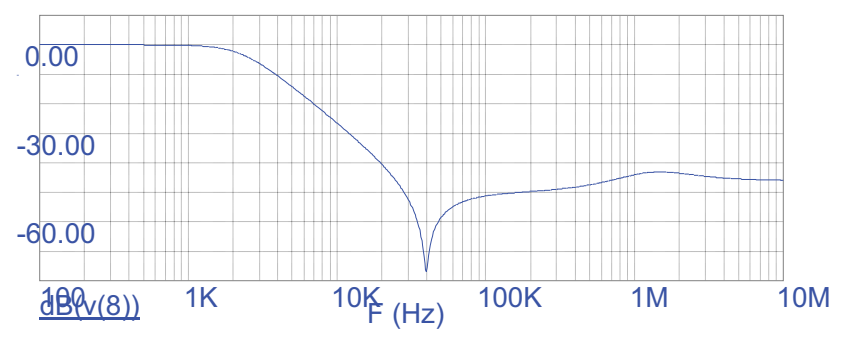

Figure 15. Simulation results at the highest frequencies.

\section{E. Friend-Delyiannis Structure} [11].

Fig. 16 is a schematic diagram of LP-D structure

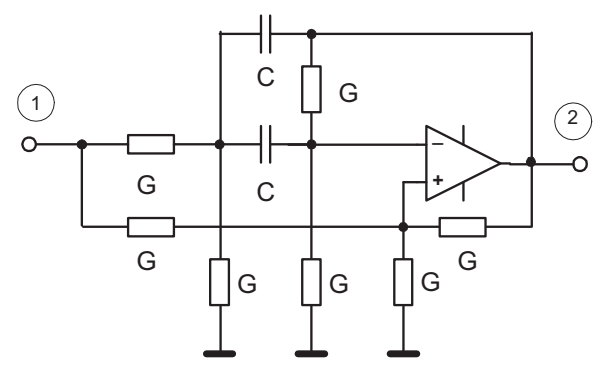

Fig.16. The structure of the Friend-Delyiannis filter structure

As we can see from Fig.17, the filter attenuation in the stopband [6] (ie. at high frequencies) does not increase to infinity, because this circuit seems as a voltage divider.

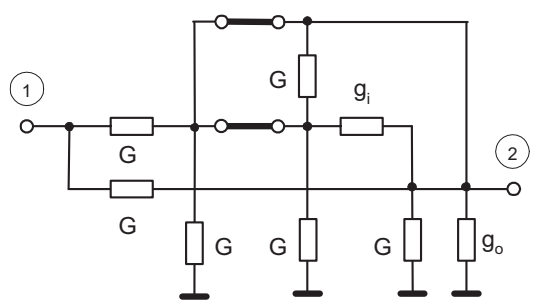

Fig.17. Model of the Friend-Delyiannis filter in high frequencies

If $\mathrm{g}_{\mathrm{o}}>\mathrm{G}>\mathrm{g}_{\mathrm{i}}$ then the output voltage $\mathrm{V}_{2}$ is given simply as (6).

$$
\mathrm{V}_{2}=\mathrm{V}_{1} \cdot \frac{\mathrm{G}}{\mathrm{G}+\mathrm{g}_{\mathrm{i}}} \neq 0
$$

i.e. is not equal to zero for higher frequencies.

\section{DISCUSSION}

Since the main function of both the anti-aliasing filter and the reconstruction filter in discrete-time signal processing (see Fig. 16) is to suppress the frequency higher than half the sampling frequency [12], it is possible, based on the theoretical analysis mentioned in paragraph II, select appropriate filter structures, taking into account the properties of real operational amplifier.

The Sallen-Key structure is suitable for the construction of the odd-order filters, while for the design of the even-order filters the Huelsmann structure is suitable.

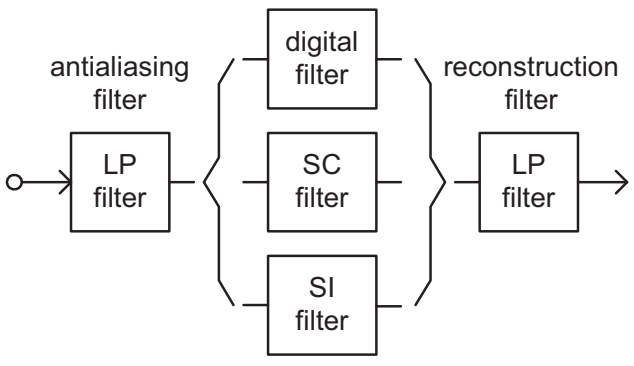

Figure 16. Antialiasing and reconstruction filer in signal processing.

In described simplified analysis (see Figs. 6, 8, 11, 14) we assumed all passive capacitors $C$ as ideal, of course. The nonzero output resistance (i.e. finite output conductance $\mathrm{g}_{\mathrm{o}}$ ) is a reason, that the filter gradually goes from "normal mode" to the frequencyindependent voltage divider.

The third-order LP-SK and LP-H biquad structures provide a monotone increasing attenuation in the stopband, i.e. in the impervious frequency band, as is depicted in Figs. 9, 12 and/or from Figs. 8, 11. The other structures discussed above do not have this advantage, therefore they are not suitable for constructing anti-aliasing and reconstruction filters.

\section{CONCLUSION}

The article compared the frequency response of the selected basic low-pass filters with a view to the decreasing of the attenuation in the stopband around to transient frequency of the used real operational amplifier. A suitable structure for a realization of a low-pass filter is recommended after analysis described above as the low-pass Huelsmann filter for even-order and Sallen-Key for odd-order anti-aliasing and/or reconstruction filters.

\section{REFERENCES}

[1] F. Yuan, A. Opal, Computer Methods for Analysis of MixedMode Switching Circuits, Kluever Academic Publisher, New York, 2004.

[2] L. Thede, Practical Analog and Digital Filtewr Design, Artech House, 2004

[3] S. Winder, Analog and Digital Filter Design, $2^{\text {nd }}$ ed, Woburn, USA, 2002, pp125-241.

[4] R. Mancini, Op Amps For Everyone - Design Reference, Texas Instruments, 2002.

[5] J. Puncochar, "Low Pass Filters Sallen and Key With Real Operational Amplifiers," Elektrorevue 10, 2005, pp. 1-13.

[6] J.Dostal, Operational Amplifiers. BEN publisher, Prague, 2006.

[7] J. Bicak, M. Leipert, M. Vlcek, A Linear Circuits an Systems, CTU publisher, Prague, 2007.

[8] T. Dostal, K. Vrba, The Electric filters, PC-DIR, Brno, 1997.

[9] D. Biolek, Solving electronic circuits. BEN publisher, Prague, 2004.

[10] W. Jung, Op Amp Applications Handbook, Elsevier, Oxford, UK, 2005, pp.307-419.

[11] P. Martinek, P. Boreš, J. Hospodka, Electrics Filters, CTU publisher, Praque, 2003.

[12] K. Hájek, J. Sedláček, Frequency Filters, Ben publisher, Prague, 2006. 